\title{
Effect of Edge Modifications to Flat Bands in Graphene Nanoribbons with Multiple Periodicity
}

\author{
Shigeo Fujimoto ${ }^{1 *}$, Ryutaro Tomita ${ }^{2}$ and Yuhei Natsume ${ }^{2}$ \\ ${ }^{1}$ Academic Link Center, Chiba University, 1-33, Yayoi-cho, Inage-ku, Chiba, 263-8522, Japan \\ ${ }^{2}$ Graduate School of Science, Chiba University, 1-33, Yayoi-cho, Inage-ku, Chiba, 263-8522, Japan \\ *e-mail: sfmt@chiba-u.jp
}

\begin{abstract}
We study the electronic states of graphene nanoribbons whose edge structures are modified in the manner that Klein's bearded bonds are periodically distributed. In the triply periodic system, complete flat bands are obtained in the case that two Klein's bonds are attached to edge per unit cell. However, there is no flat band in one Klein's bond case. We discuss the existence of flat bands in the triple periodicity system in relation to the linkage between wavefunctions; the analytic expression of the wavefunction is consecutively derived by the transfer matrix method.
\end{abstract}

Keywords: graphene, nanoriboon, flat band, transfer matrix

\section{INTRODUCTION}

Nanoelectronics based on graphene has become a fast growing field with a number of technical applications as well as fundamental studies for condensed matter physics [1-3]. In graphene nanoribbons (GNRs), the presence of edges shows characteristic electronic band structures of $\pi$-electrons [4]. Recently, researchers in the US have developed a new method to smooth out nanoribbon edges using heat treatment that makes most of resulting edges either zigzag or armchair [5]. With a transition electron aberration-corrected microscopic, dynamics for formation and stability of these two edges has been investigated [6]. In theoretical study, tuning the electronic structure of GNRs through chemical edge modification is discussed [7]. In addition, computer simulation for the mechanical tearing of graphene to form a GNR has been reported in relation with appearances of edges [8].

It is well known that zero-energy modes appear in part of the Brillouin zone (BZ) for the zigzag shaped graphene edge (zigzag edge) [9,10]. The existence of edge states is confirmed by recent experiments $[11,12]$. In addition to the zigzag edges, there is another edge of GNRs, bearded edge, which is the zigzag edge modified by attaching atomic sites having $\pi$-orbital. In this case, partial flat bands appear [13]. Because flat bands at Fermi energy give rise to large density of states, ferromagnetism originates from flat band is discussed $[9,14,15]$. In addition, transport properties are investigated in certain type of edges [16-18].

In these circumstances, much attention has been paid to relations between various types of edge structures and the existence of edge states [19-24]. The zigzag edge modified by attaching Klein's bonds in every other site possesses zero-energy mode in $2 \pi / 3 \leq\left|k a_{2}\right| \leq \pi$, where $a_{2}$ is the width of the unit cell in the doubly periodic system [22]. Quite recently, Kaneko et al. has been investigated Boron-Carbon-Nitride nanoribbons with zigzag edges using the first principles calculations [25].

It is certain that the appearance and disappearance of the flat band can be investigated by band calculations [22]. However, the theoretical understanding for the origin from the viewpoint of phase matching of the behavior has not yet been clear. In this paper, we focus on the triple periodicity with one or two Klein's bonds and investigate the appearance and disappearance of the flat band on the basis of analytic wavefunctions derived by transfer matrix method. We can make deep understanding of the linkage between wavefunctions from edges to inner sites.

\section{MODEL AND CALCULATION METHOD}

In the present paper, we discuss the system as shown in Fig. 1. The system possesses triple periodicity due to periodically distributed Klein's bonds at the edge sites.

The present Hamiltonian is expressed as the nearest-neighbor tight-binding model:

$$
H=-t \sum_{<i, j>}\left(c_{i}^{\dagger} c_{j}+\text { h.c. }\right)
$$

where $t$ is the transfer integral between nearest neighboring $i$ - and $j$-th sites while $c_{i}^{\dagger}$ and $c_{i}$ represent the creation and annihilation operators of $\pi$-electrons at $i$-th site.

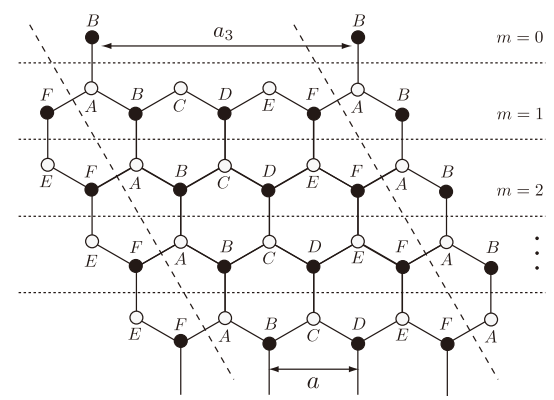

Fig. 1. The triply periodic system in which Klein's bonds are periodically attached to the edge sites. The open and filled circles represent different sublattice sites.

Figure 2 shows electronic states for triple periodicity systems with two (a) or one (b) Klein's bonds at the 
edge sites. A flat band in a whole region of $1 \mathrm{st} \mathrm{BZ}$ in the case of two Klein's bonds, whereas there is no flat band in the one Klein's bond case. In these tight binding calculations, configurations of Klein's bonds have no influence for flat bands at $E=0$. The systems with 3 Klein's bonds and with no Klein's bond are equivalent to the bearded edge [13] and the zigzag edge, respectively $[9,10]$.

Next, we introduce the transfer matrix method to derive the analytic expression of wavefunctions in the preset system.

The Schrödinger equation of the system as shown in Fig. 1 is represented by

$$
E\left(\begin{array}{l}
\psi_{m 2} \\
\psi_{m 4} \\
\psi_{m 6} \\
\psi_{m 1} \\
\psi_{m 3} \\
\psi_{m 5}
\end{array}\right)=\left(\begin{array}{cccccc}
0 & 0 & 0 & -\eta & -\eta^{*} & 0 \\
0 & 0 & 0 & 0 & -\eta & -\eta^{*} \\
0 & 0 & 0 & -\eta^{*} & 0 & -\eta \\
-\eta^{*} & 0 & -\eta & 0 & 0 & 0 \\
-\eta & -\eta^{*} & 0 & 0 & 0 & 0 \\
0 & -\eta & -\eta^{*} & 0 & 0 & 0
\end{array}\right)\left(\begin{array}{c}
\psi_{m 2} \\
\psi_{m 4} \\
\psi_{m 6} \\
\psi_{m 1} \\
\psi_{m 3} \\
\psi_{m 5}
\end{array}\right)-\left(\begin{array}{c}
\psi_{m+1,1} \\
\psi_{m+1,3} \\
\psi_{m+1,5} \\
\psi_{m-1,2} \\
\psi_{m-1,4} \\
\psi_{m-1,6}
\end{array}\right),
$$

where $t=1 . \psi_{m i}$ is the wavefunction at $i$-th sites in $m$-th row, $E$ is the energy of the system, $\eta=e^{i k a_{3} / 6}$ and its complex conjugate $\eta^{*}$ are due to Bloch's phase, $a_{3}$ is a lattice constant in the triply periodic system, and $k$ is wave number. The direction of the wave number is parallel to the edge of GNRs.

We define three-component vectors

$$
\Psi_{m}={ }^{t}\left(\psi_{m A}, \psi_{m C}, \psi_{m E}\right) \quad, \quad \Phi_{m}={ }^{t}\left(\psi_{m B}, \psi_{m D}, \psi_{m E}\right),
$$

and a $3 \times 3$ matrix

$$
S=\left(\begin{array}{ccc}
-\eta & -\eta^{*} & 0 \\
0 & -\eta & -\eta^{*} \\
-\eta^{*} & 0 & -\eta
\end{array}\right),
$$

then eq. (2) can be rewritten as follows:

$$
E\left(\begin{array}{c}
\Phi_{m} \\
\Psi_{m}
\end{array}\right)=\left(\begin{array}{cc}
\mathbf{0}_{3} & S \\
S^{\dagger} & \mathbf{0}_{3}
\end{array}\right)\left(\begin{array}{c}
\Phi_{m} \\
\Psi_{m}
\end{array}\right)-\left(\begin{array}{c}
\Psi_{m+1} \\
\Phi_{m-1}
\end{array}\right),
$$

where $\mathbf{0}_{3}$ is the null matrix of size 3 . By the use of following matrix consists of $\mathbf{0}_{3}$ and the identity matrix $\mathbf{1}_{3}$

$$
\sigma_{3 x}=\left(\begin{array}{ll}
\mathbf{0}_{3} & \mathbf{1}_{3} \\
\mathbf{1}_{3} & \mathbf{0}_{3}
\end{array}\right)
$$

we obtain the recursion relation between $(m \pm 1)$-th and $m$-th row wavefunctions
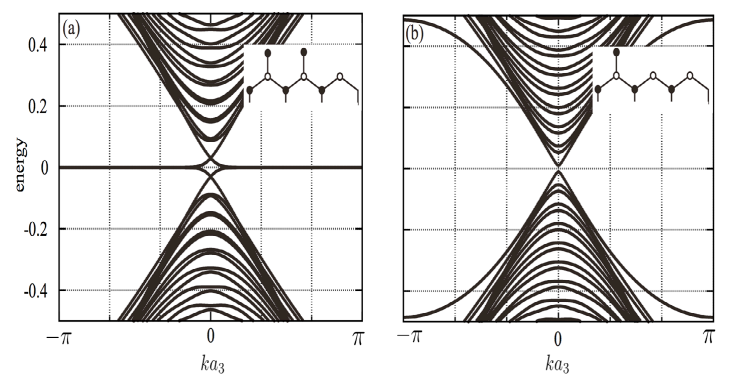

Fig. 2. Band structures for triple periodicity. (a) For a case of two Klein's bonds, a complete flat band appears at $E=0$ in a whole region of $1 \mathrm{st} \mathrm{BZ}$. (b) There is no flat band for one Klein's bond.

$$
\left(\begin{array}{c}
\Psi_{m+1} \\
\Phi_{m-1}
\end{array}\right)=\left[\left(\begin{array}{cc}
S & \mathbf{0}_{3} \\
\mathbf{0}_{3} & S^{\dagger}
\end{array}\right)-E \sigma_{3 x}\right]\left(\begin{array}{c}
\Psi_{m} \\
\Phi_{m}
\end{array}\right) .
$$

The appearance of flat bands in ribbons with zigzag edges is originated from the chiral symmetry in bulk system (graphene). This localized state is specialized by topological property of the present system. In other words, these zero-modes are dominated by the "bulkedge correspondence" and survive in finite region of one dimensional BZ for the direction of edges (perpendicular to inner sites of ribbons). In such states, wavefunctions form propagating waves from edges to inner sites instead of standing waves which generally appear in band formation for bonding-antibonding splitting by transfer energies between nearest-neighbor sites. Thus, only one sublattice is selected in accordance with the number of Klein's bonds at edges. In short, the phase matching under the Bloch condition plays an important role so as to vanish the amplitude of wavefunction at sites in another sublattice. The problem which sublattice is selected according as the number of bearded bonds in the unit cell for the direction of edges can be explained by the degree of freedom of phase matching for cases of triple periodicity as discussed from now on. However, this puzzle has not yet solved in general cases. Then, we adopt the ansatz to the selection of sublattice on the basis of results of our numerical work [22].

Especially, when $E=0$, we get the recursion relation between the wavefunctions of the $m$-th and $(m+1)$-th rows expressed as follows:

$$
\left(\begin{array}{c}
\Psi_{m+1} \\
\Phi_{m+1}
\end{array}\right)=\left(\begin{array}{cc}
S & \mathbf{0}_{3} \\
\mathbf{0}_{3} & T
\end{array}\right)\left(\begin{array}{l}
\Psi_{m} \\
\Phi_{m}
\end{array}\right)
$$

where

$$
T=\left(S^{\dagger}\right)^{-1}=\frac{-1}{2 \cos \left(k a_{3} / 2\right)}\left(\begin{array}{ccc}
\eta^{* 2} & \eta^{2} & -1 \\
-1 & \eta^{* 2} & \eta^{2} \\
\eta^{2} & -1 & \eta^{* 2}
\end{array}\right)
$$

is the transfer matrix in the sublattice consists of filled circles shown in Fig. 1 , whereas $S$ is the one in the sublattice formed from open circles.

As shown in eq. (8), because $\Psi_{m}$ and $\Phi_{m}$ are decoupled at $E=0$, each sublattice can be treated independently. 
Accordingly, at $E=0$, two types of conditions are obtained. One is "bearded-type state" expressed as $\Phi_{m}$, i.e.

$$
E=0, \Psi_{m}=0,
$$

another is "Fujita-type state" $\Psi_{m}$;

$$
E=0, \Phi_{m}=0 .
$$

As a result, the wave functions between $(m+1)$-th and edge sites are expressed as a recurrence relation

$$
\left(\begin{array}{c}
\Psi_{m+1} \\
\Phi_{m+1}
\end{array}\right)=\left(\begin{array}{cc}
S^{m} & \mathbf{0}_{3} \\
\mathbf{0}_{3} & T^{m+1}
\end{array}\right)\left(\begin{array}{c}
\Psi_{1} \\
\Phi_{0}
\end{array}\right) .
$$

\section{RESULTS AND DISCUSSION}

We discuss the analytical expression of the wavefunction for the system with triple periodicity using results obtained in the previous section.

The wavefunctions of inner sites are sequentially constructed from edge sites as in eq. (12), where $S^{m}$ and $T^{m}$ are described by

$$
S^{m}=\frac{1}{3}\left(T^{m}\right) \quad\left(\begin{array}{ccc}
\varepsilon_{1}^{m}+\varepsilon_{2}^{m}+\varepsilon_{3}^{m} & \varepsilon_{1}^{m}+e^{-i 2 \pi / 3} \varepsilon_{2}^{m}+e^{i 2 \pi / 3} \varepsilon_{3}^{m} & \varepsilon_{1}^{m}+e^{i 2 \pi / 3} \varepsilon_{2}^{m}+e^{-i 2 \pi / 3} \varepsilon_{3}^{m} \\
\varepsilon_{1}^{m}+e^{i 2 \pi / 3} \varepsilon_{2}^{m}+e^{-i 2 \pi / 3} \varepsilon_{3}^{m} & \varepsilon_{1}^{m}+\varepsilon_{2}^{m}+\varepsilon_{3}^{m} & \varepsilon_{1}^{m}+e^{-i 2 \pi / 3} \varepsilon_{2}^{m}+e^{i 2 \pi / 3} \varepsilon_{3}^{m} \\
\varepsilon_{1}^{m}+e^{-i 2 \pi / 3} \varepsilon_{2}^{m}+e^{i 2 \pi / 3} \varepsilon_{3}^{m} & \varepsilon_{1}^{m}+e^{i 2 \pi / 3} \varepsilon_{2}^{m}+e^{-i 2 \pi / 3} \varepsilon_{3}^{m} & \varepsilon_{1}^{m}+\varepsilon_{2}^{m}+\varepsilon_{3}^{m}
\end{array}\right),
$$

where $\varepsilon_{i}$ means eigenvalues of transfer matrices $S$ and $T$. For $S$, we obtain

$\xi_{1}=-2 \cos \left(k a_{3} / 6\right), \quad \xi_{2}=2 e^{-i 2 \pi / 3} \sin \left(k a_{3} / 6+\pi / 6\right), \quad \xi_{3}=2 e^{-i \pi / 3} \sin \left(k a_{3} / 6-\pi / 6\right)$

On the other hand, for $T$, we get

$\lambda_{1}=\frac{-1}{2 \cos \left(k a_{3} / 6\right)}, \quad \lambda_{2}=e^{-i 2 \pi / 3} \frac{2 \sin \left(k a_{3} / 6-\pi / 6\right)}{1-2 \cos \left(k a_{3} / 3\right)}, \lambda_{3}=e^{-i \pi / 3} \frac{2 \sin \left(k a_{3} / 6+\pi / 6\right)}{1-2 \cos \left(k a_{3} / 3\right)}$

Using eq. (14) and eq. (15), we get

$$
\lambda_{j} \xi_{j}=e^{i 2 \pi(j-1) / 3}, \quad j=1,2,3
$$

More generally, the transfer matrix $T$ is rewritten as

$$
T=\left(S^{\dagger}\right)^{-1}=(S P)^{-1}=(P S)^{-1},
$$

where

$$
P=\left(\begin{array}{lll}
0 & 0 & 1 \\
1 & 0 & 0 \\
0 & 1 & 0
\end{array}\right)
$$

is circulant matrix whose eigenvalues are $e^{i 2 \pi(j-1) / 3}$, where $j=1,2,3$. It is noteworthy that unitary matrices for $S$ and $T$ are same as ones for $P$ and $P^{-1}$, though eigenvalues are different from each other. Therefore, we get the relation shown in eq. (16).

Absolute values of eigenvalues for transfer matrices are given in Fig. 3, where the dependences on wavenumber $k$ in 1 st BZ are shown. The regions in which eigenvalues are more than 1 mean the divergence of charge densities described by wavefunctions for carbon sites. However, such divergences can be suppressed in consideration of phase matching of wavefunctions at edge sites for $m=0$ in bearded-type states $\Phi_{m}$ and for $m=1$ in Fujita-type states $\Psi_{m}$. Up to now, we would like to remark the relationship between numbers of eigenvalues exceeding 1 and degrees of freedom for wavefunctions at edge sites in each state.
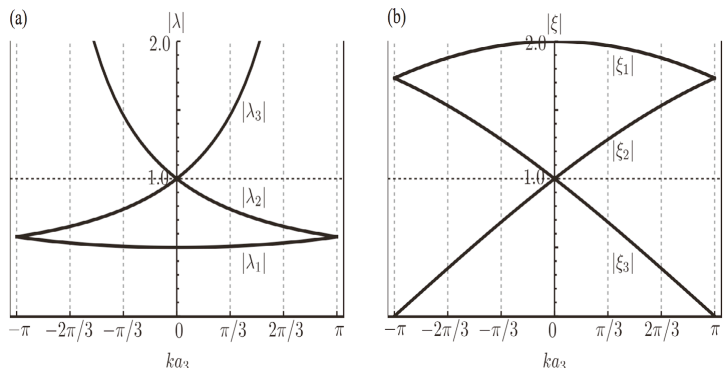

Fig. 3. The absolute values of the eigenvalue for (a) bearded-type states $\Phi_{m}$ and (b) Fujita-type states $\Psi_{m}$ in the triply periodic system.

\subsection{Case of two Klein's bonds}

As discussed in sec. 1, the edge states strongly tend to survive, because the chiral symmetry originating from Dirac points plays the essential role. In fact, energy levels at $E=0$, show no bonding-antibonding splitting as shown in Fig. 2(a); The corresponding wavefunctions make propagating waves instead of standing waves. In particular, we would like to point out that amplitudes of such the propagating waves decrease with increasing the depth from edges to inner sites of ribbon. Namely, those have behavior for convergence.

There are two types of zero-energy states as shown in sec. 2. A realized state is determined by the condition that the wavefunction converges for sufficiently inner sites from the edge. In the case that two Klein's bonds are attached to edges of triple periodicity systems, the bearded-type state is realized as will be shown later. Therefore, we should take the condition;

$$
E=0, \psi_{m A}=\psi_{m C}=\psi_{m E}=0,
$$

where $m$ is arbitrary.

Here we should pay attention to the configuration of Klein's bonds because two configurations are possible in the manner of attachment of Klein's bonds in the present case. However, the occurrence of flat bands is determined by the number of Klein's bonds in spite of the position of ones. As a result, we can take the wavefunction at 0 -th site as $\Phi_{0}={ }^{t}\left(\psi_{0 B}, \psi_{0 D}, 0\right)$, where $\psi_{0 B}$ and $\psi_{0 D}$ are determined under convergence and normalization conditions.

The wavefunction in $m$-th row can be constructed 
from the wavefunction at 0 -th row as $\Phi_{m}=T^{m} \Phi_{0}$. The absolute values of eigenvalues for $T$ are shown in Fig. 3 (a). Here, $\left|\lambda_{3}\right|\left(\left|\lambda_{2}\right|\right)$ is greater than 1 in the region for $k>$ $0(k<0)$. Therefore, we obtain the relation of $\psi_{0 D}=e^{ \pm i \pi / 3} \psi_{0 B} \quad$ considering that the coefficient of $\lambda_{3}$ for $k$ $>0\left(\lambda_{2}\right.$ for $\left.k<0\right)$ should be 0 in order to suppress the divergence of charge density $\left|\Phi_{m}\right|^{2}$ with increasing $m$.

As a result, the wavefunction is written by

$$
\Phi_{m}=\frac{e^{i \pi / 6}}{\sqrt{6}}\left(\begin{array}{c}
\lambda_{1}^{m}+e^{-i \pi / 3} \lambda_{2(3)}^{m} \\
\lambda_{1}^{m}+e^{i \pi / 3} \lambda_{2(3)}^{m} \\
\lambda_{1}^{m}-\lambda_{2(3)}^{m}
\end{array}\right) \text { for } k>0(k<0) .
$$

In fact, we obtain the characteristic behavior of charge density at $k a_{3}=0, \pi / 3$, and $2 \pi / 3$ as shown in Fig. 4. This result shows that the flat band appears in the whole BZ, which is consistent with the tight-binding calculation in Fig. 2(a). The charge density at the Dirac point becomes constant at the deep inside from the edge. On the other hand, charge densities at the wavenumbers different from the Dirac Point quickly decrease with increasing $m$.

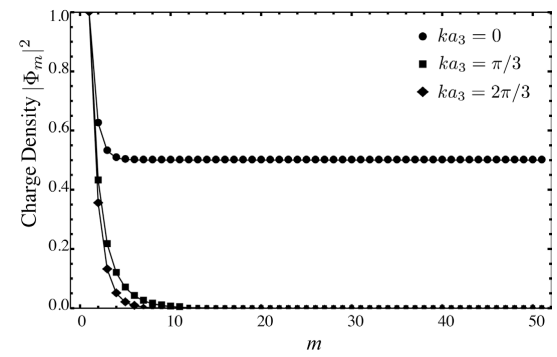

Fig. 4. Plot for charge density at $k a_{3}=0, \pi / 3$, and $2 \pi / 3$.

\subsection{Case of one Klein's bond}

In the present case, there is no degree of freedom originates from the phase part between wavefunctions at $m=0$ because of single Klein's bond. Therefore, the bearded-type state is constructed only at $k=0$ as shown in Fig. 3 (a).

On the other hand, we have a possibility for appearing the Fujita-type state. As shown in Fig. 3 (b), two absolute value of eigenvalues are greater than 1 in the whole region. In this case, however, the wavefunction cannot be constructed even if we adopt the Fujita-type state, because the phase matching is impossible for the lack of degrees of freedom between wavefunctions at $m=0$. As a result, the triple periodicity system with one Klein's bond has no flat band.

\section{SUMMARY}

We have investigated the existence of flat bands in the triple periodicity system. We showed that the behavior of flat band in connection with the phase matching between wavefunctions; the analytic expression of the wavefunction is consecutively derived by the transfer matrix method. In result, we elucidated the formation process of edge states originating from the phase matching.

We hope that the present work will give clue to future experimental investigation of graphene ribbons with various kinds of edge modifications.

\section{REFERENCES}

[1] K. S. Novoselov, A. K. Geim, S. V. Morozov, D. Jiang, Y. Zhang, S. V. Dubonos, I. V. Grigorieva, and A. A. Firsov, Science 306, 666 (2004).

[2] K. S. Novoselov, A. K. Geim, S. V. Morozov, D. Jiang, M. I. Katsnelson, I. V. Grigorieva, S. V. Dubonos, and A. A. Firsov, Nature 438, 197 (2005).

[3] Mikahail I. Katsnelson, "Grapnehe Carbon in Two Dimensionas", Cambridge University Press, New York (2012).

[4] A. H. Castro Neto, F. Guinea, N. M. Peres, K. S. Novoselov, and A. K. Geim, Rev. Mod. Phys. 81, 109 (2009).

[5] X. Li, X. Wang, L. Zhang, S. Lee, and H. Dai, Science 319, 29 (2008).

[6] C. O. Girit, J. C. Meyer, R. Erni, M. D. Rossell, C. Kisielowski, L. Yang, C.-H. Par, M. F. Crommie, M. L. Cohen, S. G. Louie, and A. Zettl, Science 323, 1705 (2009).

[7] Z. F. Wang, Q. Li, H. Zheng, H. Ren, H. Su, Q. W. Shi, and J. Chen: Phys. Rev. B 75, 113406 (2007).

[8] T. Kawai, S. Okada, Y. Miyamoto, and H. Hiura, Phys. Rev. B 80, 033401 (2009).

[9] M. Fujita, K. Wakabayashi, K. Nakada, and K. Kusakabe, J. Phys. Soc. Jpn. 65, 1920 (1996).

[10] K. Nakada, M. Fujita, G. Dresselhaus, and M. S. Dresselhaus, Phys. Rev. B 54, 17954 (1996).

[11] Y. Kobayashi, K. Fukui, T. Enoki, K. Kusakabe, and Y. Kaburagi, Phys. Rev. B 71, 193406 (2005).

[12] Y. Niimi, T. Matsui, H. Kambara, K. Tagami, M. Tsukada, and H. Fukuyama, Phys. Rev. B 73, 085421 (2006).

[13] D. J. Klein, Chem. Phys. Lett. 217, 261 (1994).

[14] K. Kusakabe and M. Maruyama, Phys. Rev. B 67, 092406 (2003).

[15] M. Maruyama and K. Kusakabe, J. Phys. Soc. Jpn. 73, 656 (2004).

[16] K. Wakabayashi and M. Sigrist, Phys. Rev. Lett. 84, 3390 (2000).

[17] K. Wakabayashi, Phys. Rev. B 64, 125428 (2001).

[18] A. Cresti and S. Roche, Phys. Rev. B 79, 233404 (2009).

[19] M. Ezawa, Phys. Rev. B 73, 045432 (2006).

[20] T. Wassmann, A. P. Seitsonen, A. M. Saitta, M. Lazzeri, and F. Mauri, Phys. Rev. Lett. 101, 096402 (2008).

[21] A. R. Akhmerov and C. W. J. Beenakker, Phys. Rev. B 77, 085423 (2008).

[22] K. Wakabayashi, S. Okada, R. Tomita, S. Fujimoto, and Yuhei Natsume, J. Phys. Soc. Jpn. 79, 03476 (2010); R. Tomita, S. Fujimoto, and Yuhei Natsume, J. Phys. Soc. Jpn. 79, 097001 (2010).

[23] W. Jaskólski, A. Ayuela, M. Pelc, H. Santos, and L. Chico, Phys. Rev. B 83, 235424 (2011).

[24] P. Delplace, D. Ullmo, and G. Montambaux, Phys. Rev. B 84, 195452 (2011).

[25] T. Kaneko, K. Harigaya, and H. Imamura, J. Phys. Soc. Jpn. 82, 083710 (2013).

\section{Note Added in Proof}

Quite recently, T. Kaneko and K. Harigaya have discussed partially flat band states in GNRs with B and $\mathrm{N}$ doing at edge atoms by using transfer matrix method in arxiv:1305.5137.

(Received May 8, 2013; Accepted October 7, 2013) 